\title{
ARTICLE OPEN \\ Patterns of cell cycle checkpoint deregulation associated with intrinsic molecular subtypes of human breast cancer cells
}

Jacquelyn J. Bower ${ }^{1,2}$, Leah D. Vance ${ }^{1}$, Matthew Psioda ${ }^{3}$, Stephanie L. Smith-Roe ${ }^{1,6}$, Dennis A. Simpson ${ }^{1}$, Joseph G. Ibrahim ${ }^{2,3,4}$, Katherine A. Hoadley ${ }^{2,5}$, Charles M. Perou ${ }^{1,2,4,5}$ and William K. Kaufmann ${ }^{1,2,4}$

Genomic instability is a hallmark of breast cancer, contributes to tumor heterogeneity, and influences chemotherapy resistance. Although Gap 2 and mitotic checkpoints are thought to prevent genomic instability, the role of these checkpoints in breast cancer is poorly understood. Here, we assess the Gap 2 and mitotic checkpoint functions of 24 breast cancer and immortalized mammary epithelial cell lines representing four of the six intrinsic molecular subtypes of breast cancer. We found that patterns of cell cycle checkpoint deregulation were associated with the intrinsic molecular subtype of breast cancer cell lines. Specifically, the luminal B and basal-like cell lines harbored two molecularly distinct Gap 2/mitosis checkpoint defects (impairment of the decatenation Gap 2 checkpoint and the spindle assembly checkpoint, respectively). All subtypes of breast cancer cell lines examined displayed aberrant DNA synthesis/Gap 2/mitosis progression and the basal-like and claudin-low cell lines exhibited increased percentages of chromatid cohesion defects. Furthermore, a decatenation Gap 2 checkpoint gene expression signature identified in the cell line panel correlated with clinical outcomes in breast cancer patients, suggesting that breast tumors may also harbor defects in decatenation Gap 2 checkpoint function. Taken together, these data imply that pharmacological targeting of signaling pathways driving these phenotypes may lead to the development of novel personalized treatment strategies for the latter two subtypes which currently lack targeted therapeutic options because of their triple negative breast cancer status.

npj Breast Cancer (2017)3:9; doi:10.1038/s41523-017-0009-7

\section{INTRODUCTION}

Cellular division is controlled by a tightly regulated process that requires accurate separation of sister chromatids upon the completion of DNA replication in order to produce two genetically identical daughter cells. The regulatory signals that control cell division are collectively referred to as the cell cycle, which is comprised of five distinct phases: quiescence $\left(G_{0}\right)$, Gap $1\left(G_{1}\right)$, DNA replication/synthesis (S), Gap $2\left(G_{2}\right)$, and mitosis (M) (Fig. 1). Transitions between different phases of the cell cycle are induced via oscillating levels of cyclins and cyclin-dependent kinases (cdks); each phase of the cell cycle is characterized by the formation of specific complexes of cyclin/cdk heterodimers.

Intracellular and/or external stimuli can halt progression of the cell cycle through a complex network of signaling events that interfere with cyclin/cdk activities controlling cell cycle progression. This pause in cell cycle progression is often referred to as a "checkpoint" and allows the cell time to repair damaged DNA or acquire sufficient levels of growth factors before transitioning to the next phase; if the DNA damage is too severe to repair, the cell may activate apoptotic signaling cascades to prevent the transmission of damaged DNA to its daughter cells. Thus, cell cycle checkpoints ensure ordered progression of the cell cycle, are critical for maintaining genomic stability, act as barriers to carcinogenesis, and are often deregulated in tumors. ${ }^{1-3}$
At least four cell cycle checkpoints may be deregulated in cancer cells: the restriction point $\left(G_{0} / G_{1}\right)$, the $G_{1}$ checkpoint, the $G_{2}$ checkpoint, and the mitosis-associated spindle assembly checkpoint (SAC). The $G_{0} / G_{1}$ restriction point is the point in $G_{1}$ at which the withdrawal of growth factors no longer induces reversion to a quiescent state; thus, it controls the cell's commitment to division. ${ }^{4}$ The restriction checkpoint is largely controlled by the $\mathrm{Rb} / \mathrm{E} 2 \mathrm{~F}$ signaling pathway: release of E2F transcription factors from $\mathrm{Rb}$ allows E2F to transcriptionally activate genes that promote the initiation of DNA replication and $S$ phase entry. Both overexpression of upstream regulators of $\mathrm{Rb}$ (such as Cyclin D1/cdk4/ 6) or inactivating mutations in the $R B 1$ gene can degrade this checkpoint and lead to early activation of DNA replication. 5,6 The $G_{1}$ DNA damage checkpoint delays the initiation of DNA replication in the presence of DNA damage and is largely controlled by the p53/p21/Mdm2 pathway.7, 8 Loss of this checkpoint can often occur via TP53 mutations or inactivation of wild-type p53 by viral proteins and lead to error-prone DNA synthesis due to the diminished amount of time allotted for DNA repair. ${ }^{9}, 10$

Two types of $G_{2}$ checkpoint responses have been previously identified: the DNA damage $\mathrm{G}_{2}$ checkpoint and the decatenation $\mathrm{G}_{2}$ checkpoint. ${ }^{11-13}$ The DNA damage $\mathrm{G}_{2}$ checkpoint delays the initiation of mitosis upon DNA damage by sequestering inactive

\footnotetext{
${ }^{1}$ Department of Pathology and Laboratory Medicine, University of North Carolina at Chapel Hill, Chapel Hill, NC 27599, USA; ${ }^{2}$ Lineberger Comprehensive Cancer Center, University of North Carolina at Chapel Hill, Chapel Hill, NC 27599, USA; ${ }^{3}$ Department of Biostatistics, University of North Carolina at Chapel Hill, Chapel Hill, NC 27599, USA; ${ }^{4}$ Center for

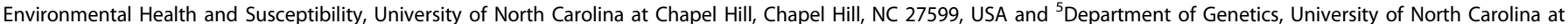
Chapel Hill, Chapel Hill, NC 27599, USA

Correspondence: Jacquelyn J. Bower (jjbower@email.unc.edu)

${ }^{6}$ Present address: Division of the National Toxicology Program, National Institute of Environmental Health Sciences, 111 T.W. Alexander Drive, National Institutes of Health, Research Triangle Park, NC 27709, USA
}

Received: 15 August 2016 Accepted: 7 February 2017

Published online: 31 March 2017 
Mitotic Index (\% of Cells in Mitosis)

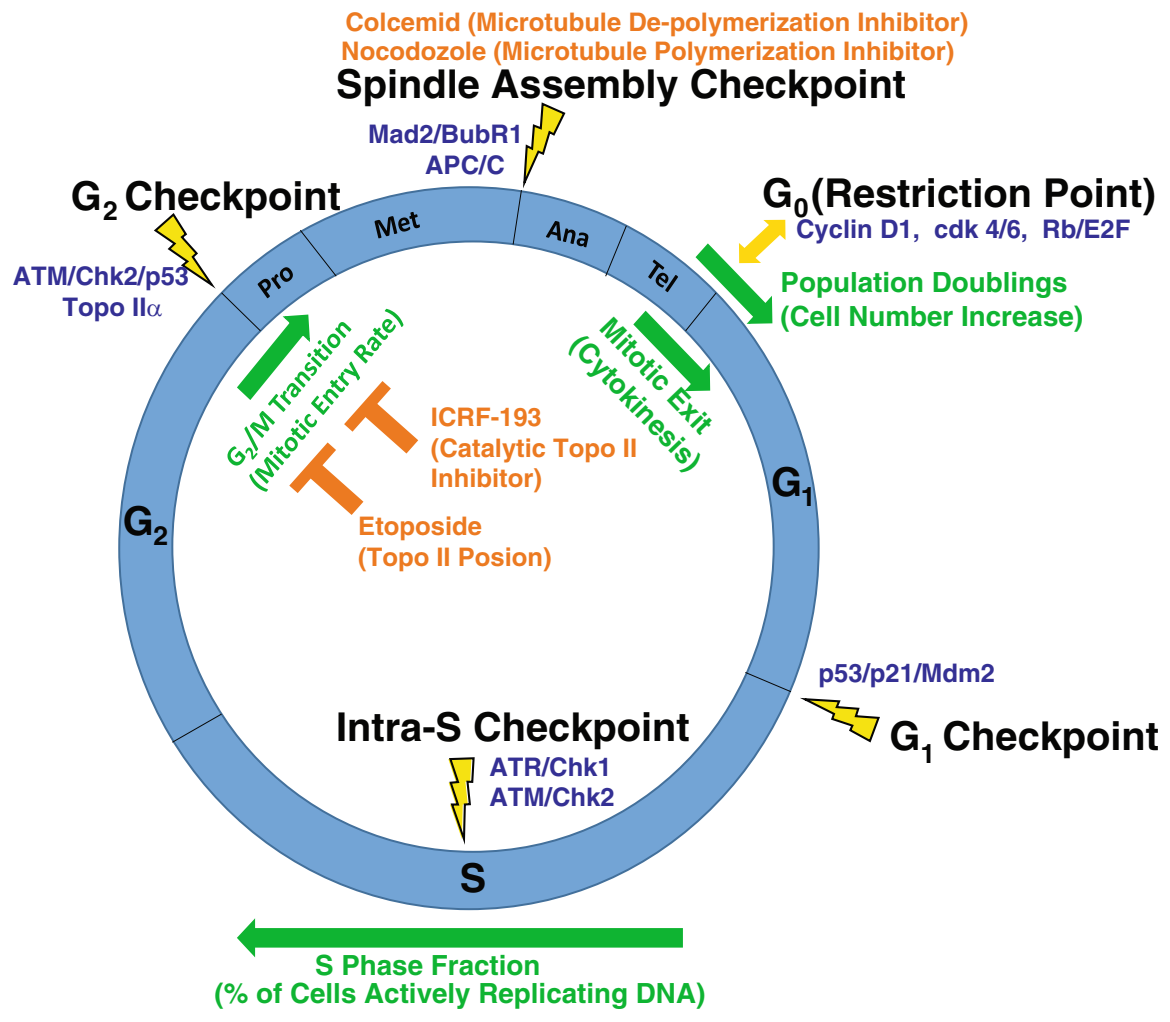

\begin{tabular}{l}
\hline \multicolumn{1}{|c|}{ LEGEND } \\
Major Checkpoint Regulators \\
Measures of Cell Proliferation \\
Drugs Inhibiting Cell Cycle Progression
\end{tabular}

Fig. 1 Diagram of cell cycle regulation. Phases of the cell cycle are shown inside the blue circle in the center of the figure $\left(G_{0}, G_{1}, S\right.$, $G_{2}$, and mitosis which consists of several sub-phases: prophase (Pro), metaphase (Met), anaphase (Ana), and telophase (Tel)). The $G_{0}$ Restriction Point is designated with a yellow dual headed arrow to illustrate the reversible nature of cell cycle entry and quiescence. As cells progress through the cycle, exogenous perturbations can activate checkpoints that arrest cells during phase transitions (checkpoints are designated by yellow lightning bolts). Several measures of cellular proliferation are shown in green and span the cell cycle phases in which these markers are present. Drugs that inhibit cell cycle progression are shown in orange with their targets and mechanisms of action designated in subsequent parentheses. Components of major regulatory pathways triggering each checkpoint are listed in dark blue font near the checkpoint in which they play a role. Precise control over the regulation of the cell cycle is a requirement for ensuring accurate DNA replication and cell division

cyclin $\mathrm{B} 1 / \mathrm{cdk} 1$ in the cytoplasm, thus preventing entry into mitosis. ${ }^{14,} 15$ The decatenation $G_{2}$ checkpoint is molecularly distinct from the DNA damage $G_{2}$ checkpoint in that it is activated in response to catalytic inhibition of topoisomerase Ila (topo Ila) without overt DNA damage. ${ }^{12,16,17}$ Major players in both the DNA damage and decatenation $G_{2}$ checkpoint include the ATM/Chk2/ p53 pathway and attenuation of either $G_{2}$ checkpoint leads to chromosomal instability. ${ }^{18}$ Finally, the SAC acts in mitosis to delay the onset of anaphase until all chromosomes exhibit bipolar attachment to the mitotic spindle. ${ }^{2,19,}{ }^{20}$ Major effectors of SAC function include $A P C / C, B u b R 1$, and Mad2, and impaired SAC signaling often leads to the formation of multipolar spindles and/ or the unequal partitioning of sister chromatids into daughter cells. ${ }^{21}$ Defects in all of these cell cycle checkpoints have been shown to play an integral role in tumor initiation and/or progression and affect the sensitivity of tumors to both cytotoxic and endocrine drugs. ${ }^{22-24}$

The role of the restriction and $G_{1}$ checkpoints in breast cancer signaling, genome maintenance, and chemosensitivity has been previously characterized; ${ }^{24,25}$ however, the relationship between $\mathrm{G}_{2}$ or SAC checkpoint function, genomic instability, and chemoresistance is poorly understood. Six intrinsic molecular subtypes of breast tumors have been proposed, ${ }^{26,}{ }^{27}$ and several studies imply that these subtypes contain similar molecular defects that contribute to disease progression and variability in chemotherapeutic response, ${ }^{28-30}$ which may confer synthetic lethality to subsets of cancer cells. ${ }^{31,32}$ Although these studies imply that genomic instability plays a role in these processes, the nature of the specific molecular defects contributing to breast cancer outcomes remains elusive. To explore the underlying mechanisms of genomic instability in breast cancer cells, $G_{2}$ and $M$ checkpoint 
functions were assessed in a panel of 24 cell lines using a flow cytometry-based mitotic entry rate (MER) assay that allows for discrimination among three molecularly distinct $G_{2} / M$ checkpoints: the DNA damage $G_{2}$ checkpoint, the decatenation $G_{2}$ checkpoint, and the SAC. The panel is comprised of cell lines representing four of the six intrinsic molecular subtypes of breast tumors: six luminal $B($ LumB), four basal-like (BL), six claudin-low $(\mathrm{CL})$, and four Her2-enriched (Her2E) cell lines. Luminal $A$ and normal-like breast cancer cell lines are unavailable, and four immortalized non-tumorigenic human mammary epithelial cell
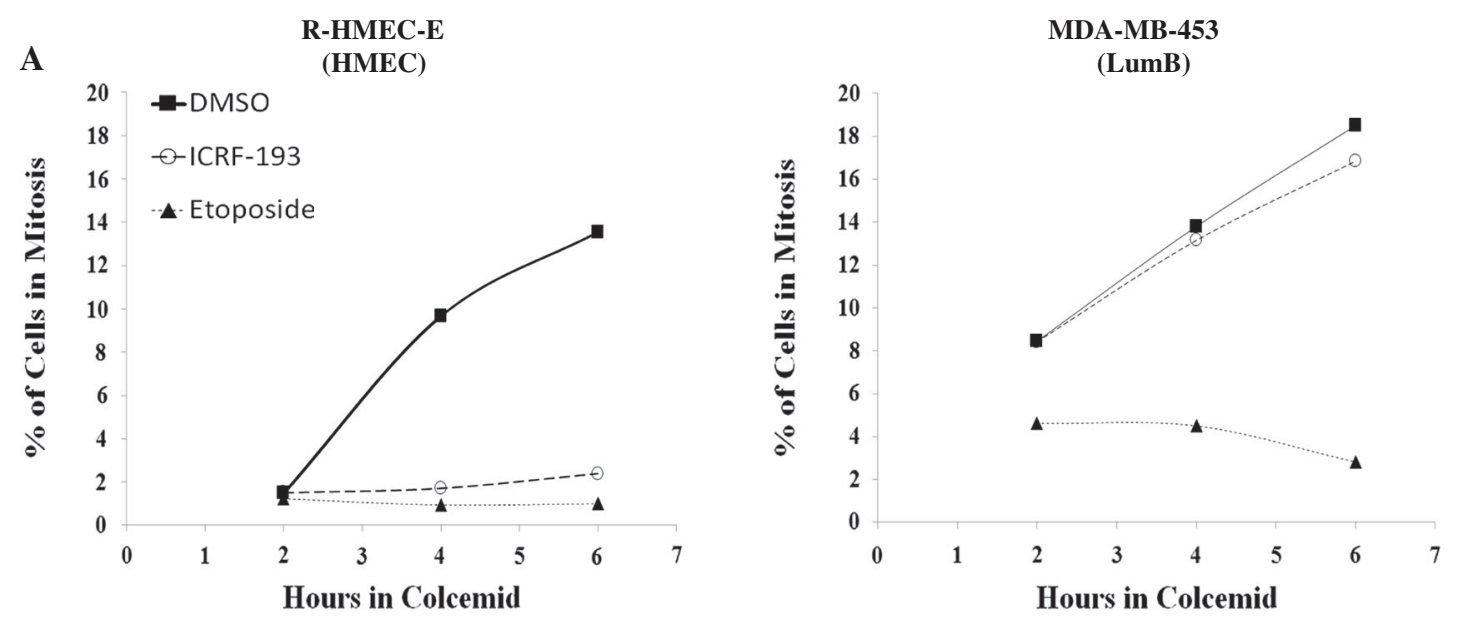

B Decatenation $\mathbf{G}_{2}$ Checkpoint (ICRF-193)

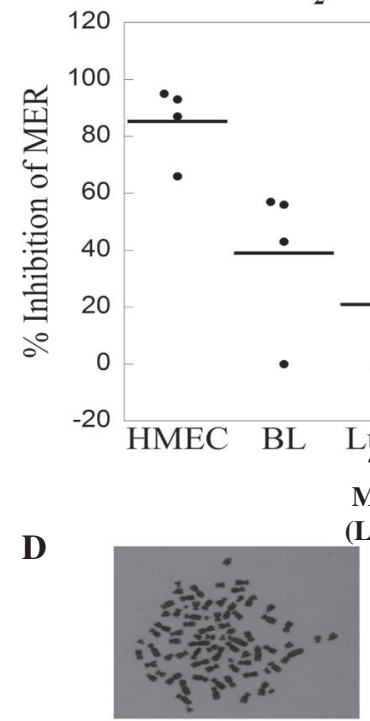

$0.1 \%$ DMSO

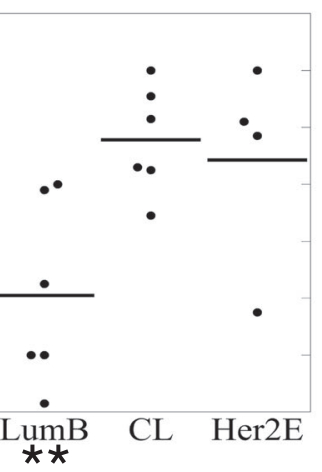

C DNA Damage $\mathbf{G}_{\mathbf{2}}$ Checkpoint (Etoposide)

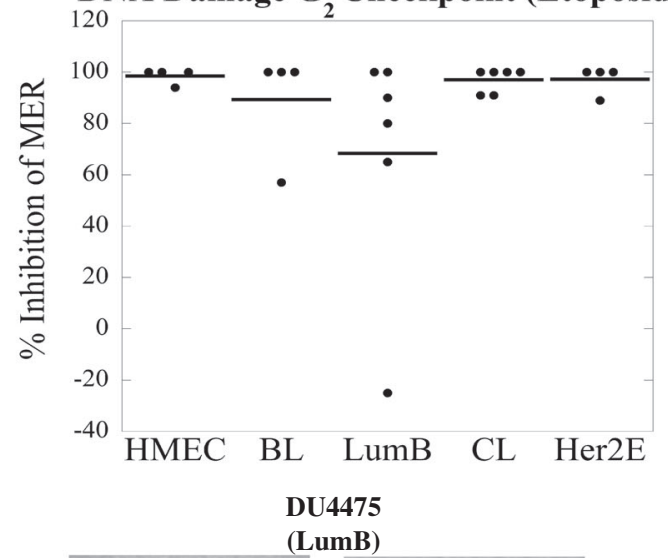

(LumB)

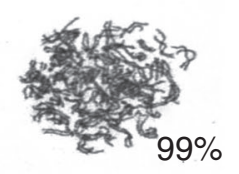

$4 \mu \mathrm{M}$ ICRF-193

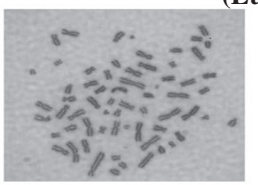

0.1\% DMSO

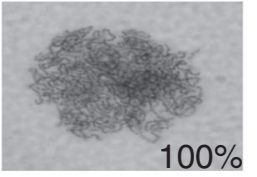

$4 \mu \mathrm{M}$ ICRF-193

HMECs

$\mathbf{E}$

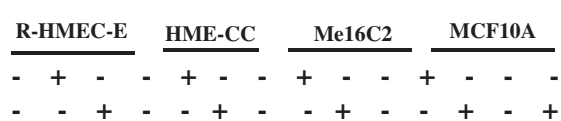

NHF1-hTERT

$4 \mu$ M ICRF-193

$4 \mu \mathrm{M}$ Etoposide

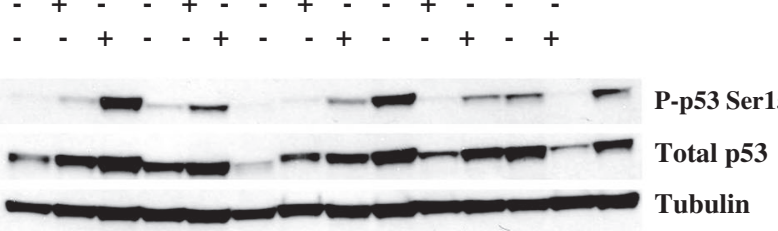

LumBs

MDA-MB-415 MCF7 ZR-75-01 MDA-MB-453 HCC1428 NHF1-hTERT

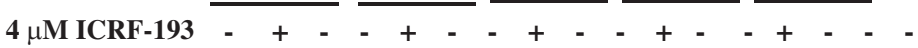

$4 \mu \mathrm{M}$ Etoposide - $-+\quad-\quad+\quad-\quad+\quad-\quad+\quad-\quad+\quad+$
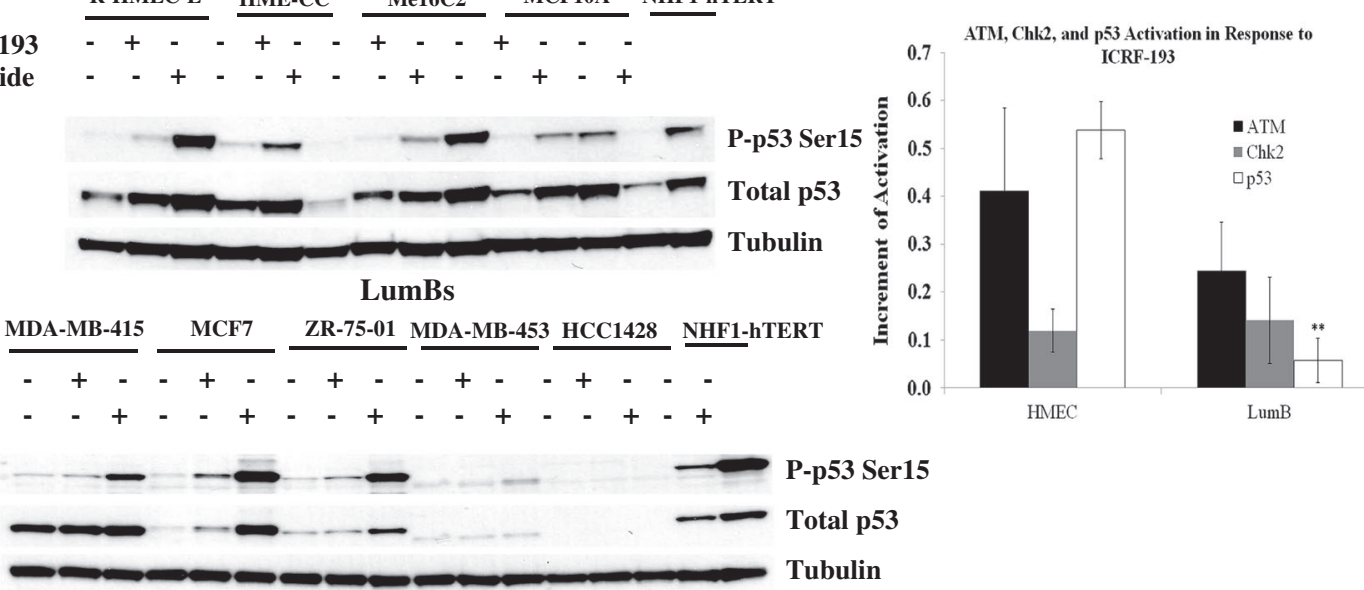
Fig. 2 The decatenation $G_{2}$ checkpoint response is impaired in luminal $B$ (LumB) breast cancer cell lines. A panel of non-tumorigenic immortalized mammary epithelial cell lines (HMEC) and breast cancer cell lines were assessed for $\mathrm{G}_{2}$ and $M$ checkpoint functions using a mitotic entry rate (MER) assay to monitor the rate of the $G_{2} / M$ transition in the presence of the topo II catalytic inhibitor ICRF-193 (decatenation $\mathrm{G}_{2}$ checkpoint) or the DNA-damaging topo II poison etoposide (DNA damage $\mathrm{G}_{2}$ checkpoint). a Example MERs of an HMEC (RHMEC-E) cell line with effective decatenation and DNA damage $G_{2}$ checkpoints and a LumB (MDA-MB-453) cell line with a defective decatenation $G_{2}$ checkpoint. $\mathbf{b}$ and $\mathbf{c}$ The average percent inhibition of the MER of each cell line grouped according to intrinsic molecular subtype. Each point on the graph represents the average of 3 independent experiments for an individual cell line, and the bold lines represent the class average. $\mathbf{d}$ Example metaphases of LumB cell lines in the presence of DMSO exhibiting individualized chromosomes. Severely undercondensed and/or entangled chromosomes were observed in $>88 \%$ of LumB cells upon treatment with $4 \mu \mathrm{M}$ ICRF-193, suggesting that ICRF193 is capable of inhibiting topoisomerase II in LumB cell lines. Percentages of entangled/under-condensed chromosomes are shown in the lower right hand corner of each ICRF-193 treated example. e HMECs activate p-Ser15 p53 after ICRF-193 or etoposide treatment (top panel). The HME-CC etoposide sample displayed aberrant mobility of ATM and reduced expression of p53 which could not be reproduced; therefore, this sample was omitted from the analysis. LumB cell lines exhibit reduced levels of p-Ser15 p53 in response to ICRF-193 (lower panel). Quantification of p53 activation is shown in the right panel. Data are representative of two independent experiments. ${ }^{*} p$-value $<0.05$, ${ }^{* *} p$ value that remains significant when controlling for FDR (5\%), BL: basal-like, CL: claudin-low, Her2E: Her2-enriched

lines (HMECs) were employed as positive controls. These cell lines recapitulate the gene expression profiles, abnormal genomic features, and phenotypes of in vivo breast tumors, ${ }^{33-35}$ and exhibit subtype-specific responses to several chemotherapeutic agents. ${ }^{32}$

Because no comprehensive functional analysis of $G_{2} / M$ checkpoints has been described for breast cancer cell lines to date and existing reports almost exclusively utilize three breast cancer cell lines (MCF7, HCC1937, and MDA-MB-231) to investigate the accumulation of cells in $\mathrm{G}_{2} / \mathrm{M}$ (but not checkpoint function), the data generated from this study provides a valuable resource for delineating the role of $\mathrm{G}_{2} / \mathrm{M}$ checkpoints in breast cancer cells. Furthermore, the findings of this study indicate that deregulation of $G_{2}$ and $M$ checkpoint functions, aberrant cell cycle regulation patterns, and chromatid cohesion defects coincide with breast cancer intrinsic molecular subtypes and that a gene signature for decatenation $G_{2}$ checkpoint function identified in the cell line panel correlates with clinical outcomes in breast cancer patients. These data collectively suggest that pharmacological targeting of pathways driving these genomic instability phenotypes may lead to the development of new personalized treatment strategies for breast cancer subtypes that currently lack targeted therapies.

\section{RESULTS}

The decatenation $\mathrm{G}_{2}$ checkpoint is impaired in LumB breast cancer cell lines

To assess $G_{2} / M$ checkpoint function, a MER assay utilizing two topo II inhibitors exhibiting distinct mechanisms of action was employed to monitor the $\mathrm{G}_{2}-\mathrm{M}$ transition rate. Cells were incubated with colcemid (to prevent mitotic exit) for 2-6h in the presence or absence of the topo II catalytic inhibitor ICRF-193 (which does not overtly damage DNA) to measure decatenation $G_{2}$ checkpoint function or the topo II poison etoposide (which induces DNA damage) to measure DNA damage $G_{2}$ checkpoint function at concentrations that arrest $98-100 \%$ of normal human diploid fibroblasts (NHF1-hTERTs) and immortalized lymphoblasts in $\mathrm{G}_{2}{ }^{36}$ The use of colcemid in the MER assay blocks cells from exiting mitosis, allowing for a strict examination of the $G_{2}$ to $M$ transition, thus minimizing any confounding effects related to the rate of mitotic exit. The MER was calculated from the linear portion of the resulting line ( $2-6 \mathrm{~h}$ time points) and is expressed as the percentage of cells entering mitosis per hour. ${ }^{36}$

MER examples are shown for the HMEC cell line R-HMEC-E in the left panel of Fig 2a. These cells accumulated in mitosis when incubated in colcemid (and the vehicle control dimethyl sulfoxide (DMSO), closed squares) reflecting their transition from $G_{2}$ to $M$; however, in the presence of the catalytic inhibitor ICRF-193 (open circles), mitotic accumulation of R-HMEC-E cells was severely inhibited suggesting that the majority of these cells activate a decatenation $\mathrm{G}_{2}$ checkpoint response. In the presence of the topo II poison etoposide, the mitotic accumulation of R-HMEC-E cells was also severely inhibited (closed triangles), indicating that this cell line exhibits an effective DNA damage $G_{2}$ checkpoint. Conversely, the LumB breast cancer cell line MDA-MB-453 continued to accumulate in mitosis in the presence of ICRF-193, but not etoposide (Fig. 2a, right panel), suggesting that a majority of these cells evaded the decatenation $\mathrm{G}_{2}$ checkpoint but arrested in $G_{2}$ upon DNA damage.

All 24 cell lines were subjected to the MER-based $G_{2}$ checkpoint assay and the averages of three independent experiments for each cell line (grouped according to molecular subtype) are shown in Fig. $2 b$ (individual cell line averages \pm SEM are provided in Table S1). As expected, all four positive control HMEC cell lines displayed severe MER inhibition in response to ICRF-193 suggesting that this class exhibits an effective decatenation $\mathrm{G}_{2}$ checkpoint. In contrast, checkpoint functions of the breast cancer cell lines were highly variable (Fig. 2b). Upon grouping the cell lines by intrinsic molecular subtype, the LumB and BL cell lines appeared to exhibit attenuated decatenation $G_{2}$ checkpoint function, whereas the HER2E and $\mathrm{CL}$ subtypes displayed checkpoint function similar to the HMECs. A set of statistical linear mixed models (LMMs) was employed to compare $G_{2}$ checkpoint functions among the different subtypes (see Supplementary Information, (SI)). These analyses established that the LumB class (but not the BL class) of breast cancer cell lines exhibited defects in decatenation $\mathrm{G}_{2}$ checkpoint function (** $p<$ 0.05 , FDR < 0.05 ). Although the distribution of $\%$ inhibition of MER in the BL cell lines initially appeared similar to that of the LumB group, closer inspection of the MER data revealed that the $B L$ group exhibited very low MERs in the absence of a topo II catalytic inhibitor, indicating that this group was not responding to colcemid treatment; thus, the percent inhibition of MER appears artificially low in the BL group.

Complementary experiments were performed to assess whether breast cancer cell lines were capable of activating the DNA damage $G_{2}$ checkpoint using a concentration of the topo II poison etoposide equivalent to that of the catalytic inhibitor ICRF$193(4 \mu \mathrm{M})$. All four HMEC cell lines displayed an effective DNA damage $G_{2}$ checkpoint in response to etoposide (Fig. 2c). The majority of the breast cancer cell lines also exhibited large MER inhibition in the presence of etoposide and no significant differences were observed for any of the breast cancer cell line classes; thus, these breast cancer cell lines were capable of activating the DNA damage $G_{2}$ checkpoint at levels comparable to those of HMECs, with the exception of the DU4475 LumB cell line (Table S1).

To confirm that ICRF-193 was able to inhibit topo II activity in the LumB cell lines, cytogenetic preparations were examined in the presence or absence of ICRF-193 and representative metaphases of two LumB cell lines are shown in Fig. $2 \mathrm{~d}$. In the presence of DMSO, the majority of the LumB metaphases exhibited individualized condensed chromosomes. However, 
upon inhibition of topo II with ICRF-193, $>88 \%$ of all LumB mitotic cells displayed chromosomes that were under-condensed and/or entangled (data not shown). Thus, the observed defect in decatenation $G_{2}$ checkpoint function was unlikely to be the result of a failure of ICRF-193 to inhibit topo II catalytic activity in the LumB class, supporting the conclusion that the defect is due to dysfunctional checkpoint activation and not an inability of the LumB class to respond to ICRF-193 treatment.

Previous studies demonstrate that $\mathrm{p} 53$ is activated in response to ICRF-193, and cell lines with defective decatenation $G_{2}$ checkpoint function exhibit attenuated activation of $\mathrm{p} 53 .^{36,}{ }^{37}$ Therefore, the LumB and HMEC classes were examined to determine whether cell lines lacking a decatenation $\mathrm{G}_{2}$ checkpoint exhibit attenuated p53 phosphorylation. To facilitate comparisons across different blots and account for exposure differences, the same NHF1-hTERT sample was loaded onto each gel and the increment of activation of $\mathrm{p}$-Ser15 p53 for each cell line was normalized to that of the NHF1-hTERT internal control. As shown in the upper panel of Fig. 2e, all four HMEC cell lines responded to ICRF-193 with induction of total p53 and p-Ser15 p53. Larger increases in p-Ser15-p53 were observed upon etoposide treatment in the majority of HMEC cell lines, signifying that activation of p53 signaling by ICRF-193 in HMECs is similar to that observed in other non-tumorigenic cell lines. ${ }^{36}$ In contrast, the LumB cell lines exhibited attenuated induction of p-Ser15 p53 in response to ICRF-193 (Fig. 2e, bottom panel). At least two of these cell lines (HCC1428 and MDA-MB-453) appeared to express low or undetectable levels of p53 despite harboring wildtype TP53 genes. ${ }^{38}$ Only the MDA-MB-415 cell line exhibited a mutant form (Y236C) and high basal levels of p53, suggesting that p53 activation was attenuated by a mechanism other than mutation in most of the LumB lines. The increment of p-Ser15 p53 upon etoposide treatment for the LumB class was not statistically different from the HMEC class (Fig. S1B), suggesting that attenuated signaling was not due to the loss of an inherent ability to phosphorylate Ser15 of p53, but rather was less responsive to topo II catalytic inhibition.

Activation of p-Ser 1981 of ATM and p-Thr 68 of Chk2 can contribute to p-Ser15 activation of $\mathrm{p} 53$ and have also been observed upon ICRF-193 exposure; ${ }^{36}$ however, no significant differences in ATM or Chk2 activation were observed in the LumB class when compared to the HMEC class, likely due to high levels of variation among cell lines (Fig. S1A-B). Western blots of all cell lines comprising the remaining intrinsic subtypes are shown in Fig. S1C$\mathrm{G}$. None of the intrinsic subtypes exhibited a statistically significant decrease in ATM or Chk2 when compared to the HMEC class upon either ICRF-193 or etoposide exposure (Fig. S1B); however, the Her2E class also exhibited attenuated activation of $\mathrm{p} 53$ in response to ICRF-193. Due to the short exposure time (3h), this may be a result of the slower growth rate of the Her2E lines as described further below. Taken together, these results indicate that the LumB class harbors a defective decatenation $\mathrm{G}_{2}$ checkpoint.

\section{Breast cancer cell lines exhibit altered $S / G_{2} / M$ cell cycle} progression

During the initial $G_{2} / M$ checkpoint screen, several cell lines exhibited a low MER (Table S1). Because interpretation of checkpoint function can be confounded by differential growth rates, the cell line panel was assessed for differential patterns of cell proliferation and/or deregulated cell cycle progression kinetics to account for inherent differences in cell cycle phase length that might interfere with interpretation of the $G_{2} / M$ checkpoint functionality experiments and to ensure that the LumB defect in decatenation $G_{2}$ checkpoint function was not attributable to inherent differences in MER. Such confounding effects have been previously reported and are often due to the dependency of some checkpoint assays on singular markers of cell proliferation. ${ }^{36}$
Three measures of cell proliferation were compared via flow cytometry including the $S$ phase fraction as determined by EdU incorporation (S), the mitotic index (MI) as measured by $\mathrm{MPM}^{+} / 4 \mathrm{~N}$ DNA content, and the MER. The number of population doublings (PDLs) occurring during continuous culture was also determined by counting the total number of cells during each passage and calculating the doubling time of each cell line (PDL/ week). In Fig. $3 a-d$, the individual averages for each cell line are depicted and grouped by molecular subtype. (Averages for each cell line \pm SEM are provided in Table S2).

In Fig. $3 a$, the $S$ phase fractions of the Her2E and LumB subtypes appeared lower than the other classes. LMM analysis supported the observation of a decrease in $\mathrm{S}$ for the Her2E class, but the decrease in LumB lines was not statistically significant suggesting that the $\mathrm{HMEC}, \mathrm{BL}, \mathrm{CL}$, and LumB classes exhibit comparable percentages of cells undergoing DNA replication. In Fig. 3b, the BL cell lines exhibited an increased MI compared to the HMECs, and LMM analysis reinforced this observation suggesting that the HMEC, CL, LumB, and Her2E classes exhibit similar Mls. In Fig. 3c, the MER of the $\mathrm{BL}, \mathrm{CL}$, and Her2E classes were lower than the HMEC class; these observations were corroborated by LMM analysis and suggest that only the HMEC and LumB classes exhibited similar MERs. In Fig. 3d, PDL measurements suggested that the LumB and Her2E subtypes have lower PDLs, and LMM analysis validated that both classes exhibited significantly lower PDLs when compared to the HMEC class. The LumB class was comparable to the HMEC class by every other measure of cell proliferation, indicating that a MER comparison of the HMEC and LumB classes was an appropriate measure of both decatenation and DNA damage $G_{2}$ checkpoint function in LumB cell lines. $\left({ }^{* *} p<\right.$ $0.05, \mathrm{FDR}<0.05$ )

Because the completion of DNA replication is coupled to the onset of mitosis, concomitant increases in multiple measures of cell proliferation (Fig. $3 a-c$ ) are expected to occur in cells that maintain strict regulatory control over $S / G_{2} / M$ cell cycle progression to preserve a diploid genome. ${ }^{39,40}$ Conversely, this relationship would be disrupted if cell cycle phase transitions were deregulated. Thus, two adjacent markers of cell cycle phase were assessed for the presence of a correlative relationship, ${ }^{41}$ and an absence of correlation between those markers would imply that the transition between those two cell cycle phases was delayed and/or aberrantly regulated.

The HMEC and LumB classes exhibited a highly correlative relationship between $S$ phase fraction and $\mathrm{Ml}(p<0.0001$ and 0.0041 , respectively, Table 1), suggesting that the cell cycle transitions among $S / G_{2} / M$ phases follows an ordered progression. However, the $\mathrm{BL}, \mathrm{CL}$, and Her2E classes exhibited no correlative relationship between $\mathrm{S}$ phase fraction and $\mathrm{Ml}$ suggesting that cell cycle progression is delayed or aberrantly regulated at some point between the initiation of DNA replication and mitotic exit. To further refine the deregulated point(s) in the cell cycle, $S$ phase fraction was compared to the MER measurement to determine if $S$ or $G_{2}$ progression was delayed. Only the HMEC class $(p=0.0006)$ exhibited a correlation between the $S$ and MER; all of the breast cancer cell line classes displayed decoupling of the $S$ phase fraction and MER suggesting that these classes may spend longer periods of time in the $\mathrm{S}$ or $\mathrm{G}_{2}$ phases, or exhibit aberrant cell cycle control during $S / G_{2}$. Finally, the HMEC, LumB, and $C L$ classes all exhibited a correlative relationship between the time-dependent MER and MI ( $p=0.012, p<0.0001$, and $p=0.0051$, respectively) suggesting that these classes maintain ordered mitotic progression. In contrast, the BL and Her2E classes showed no significant correlation between MER and MI, indicating that these classes may not respond to the microtubule polymerization inhibitor colcemid and/or exhibit difficulty completing mitosis. $\left({ }^{*} p<0.05,{ }^{* *} p<0.05\right.$, $\mathrm{FDR}<0.05$ )

Only the non-tumorigenic HMEC class exhibited a correlative relationship among all three proliferation markers, suggesting that 
regulation of the cell cycle in HMECs is linked, occurs in an ordered progression, and illustrates the tightly controlled regulation of $\mathrm{S} / \mathrm{G}_{2} / \mathrm{M}$ progression kinetics in non-tumorigenic cells. In contrast, breast cancer cell lines exhibited deregulation of one or more distinct cell cycle phases suggesting that $S / G_{2} / M$ progression was delayed or altered. The $\mathrm{BL}$ and Her2E classes displayed no correlative relationships, suggesting that multiple defects in cell cycle regulation were present. Because the data in Fig. 3a-d demonstrate that the Her2E class also exhibited lower proliferation rates in culture when compared to the HMECs, we are reluctant to make conclusions regarding cell cycle checkpoint function in cell lines of the Her2E class without additional data. In summary, these data suggest that breast cancer subtypes are associated with altered regulation of $S / G_{2} / M$ progression, and that the $B L$ and $C L$ classes may exhibit $S / G_{2} / M$ progression defects that evaded detection by the MER assay.

A

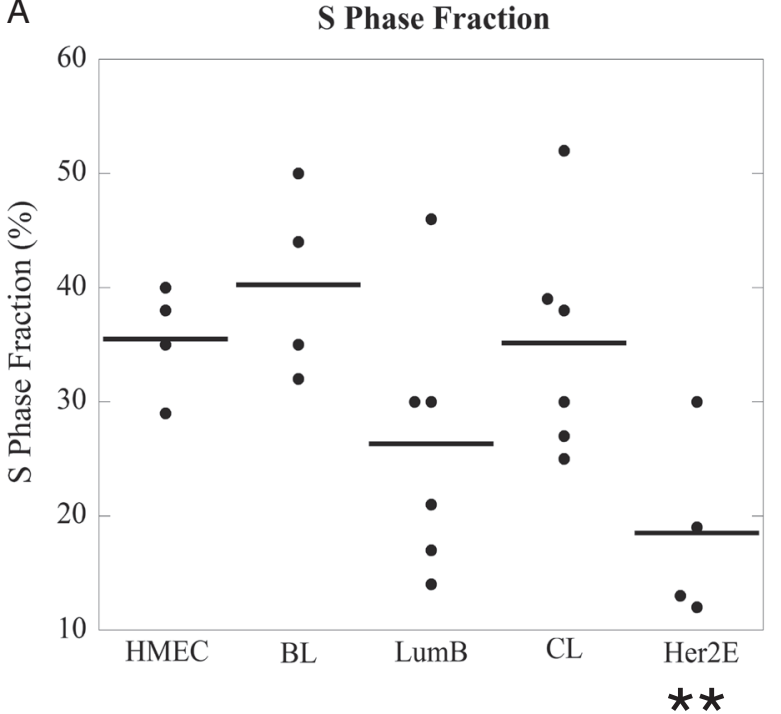

C

MER

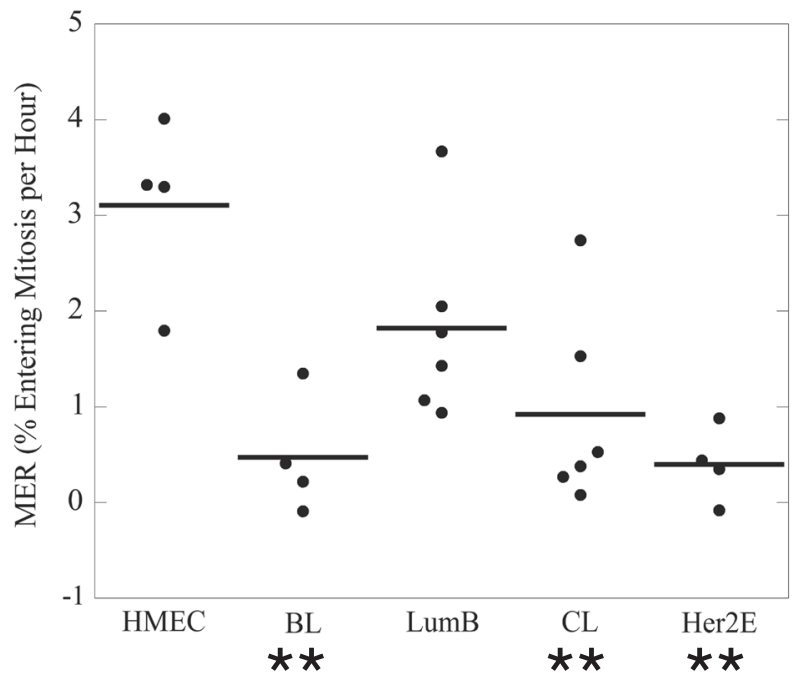

$\mathrm{CL}$ and $\mathrm{BL}$ breast cancer cell lines harbor chromatid cohesion defects; the SAC is impaired in BL breast cancer cell lines

To determine whether the $\mathrm{BL}$ and $\mathrm{CL}$ classes exhibited $\mathrm{S} / \mathrm{G}_{2} / \mathrm{M}$ checkpoint defects that were not identified by the MER screen, metaphases were analyzed to evaluate gross chromosomal structure aberrations which are often indicative of checkpoint defects. All metaphases were scored in a blind manner, and example spreads are shown in Fig. 4a for an HMEC, BL, and CL cell line. Overall, metaphases of the LumB, and Her2E classes exhibited similar probabilities of harboring a cohesion defect when compared to the HMEC class (Table S3A, $p=0.1674$ and $p=0.4743$, respectively). In contrast, the $\mathrm{CL}$ and $\mathrm{BL}$ classes appeared to exhibit an increased probability of harboring cohesion defects that ranged from mild to severe (Fig. 4a).

B

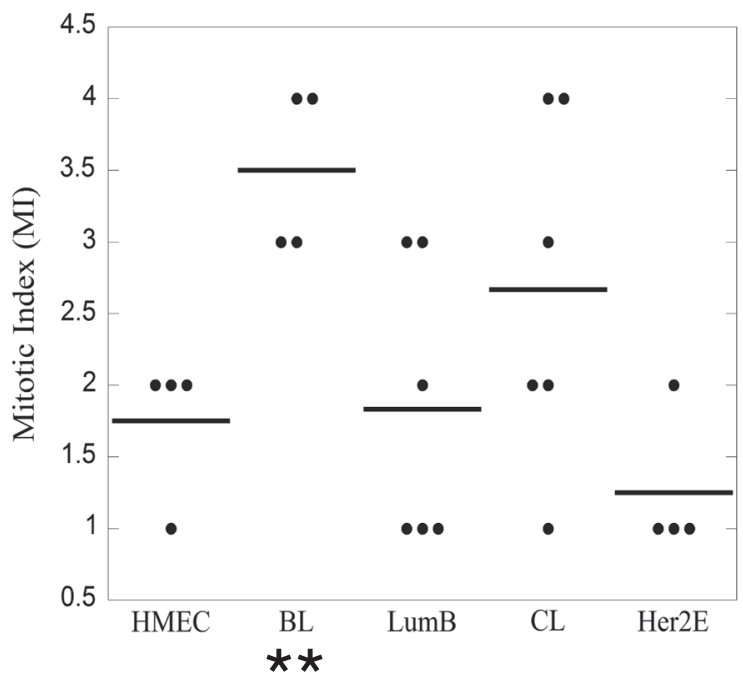

D

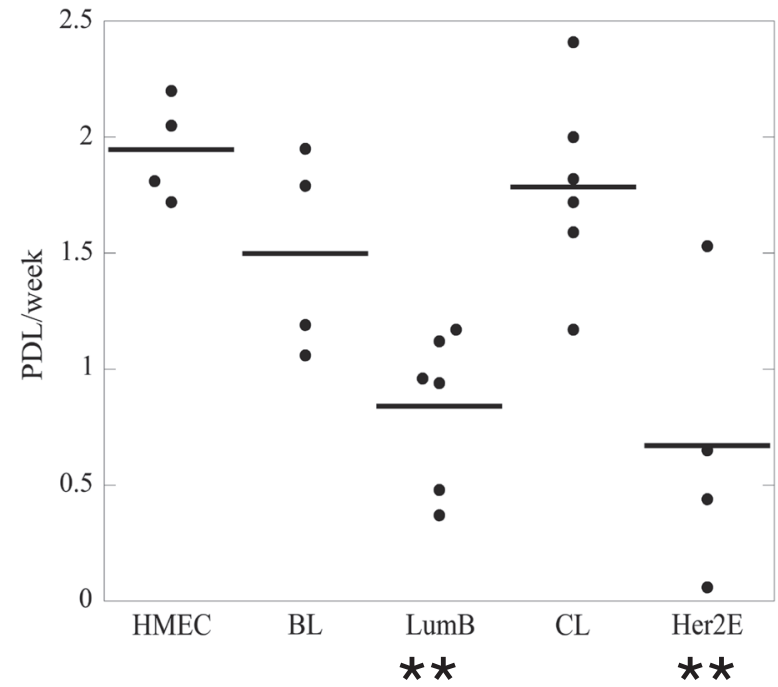

Fig. $3 \mathrm{~S} / \mathrm{G}_{2} / \mathrm{M}$ progression is aberrantly regulated in breast cancer cell lines of all classes. The average percentage of cells in $\mathrm{S}$ phase (a) or mitosis (b) and the MER (c) is shown for each cell line and grouped according to intrinsic molecular subtype. Each point represents the average of at least three independent experiments for an individual cell line, and the bold lines represent the class average. The Her2E class exhibited a lower S phase fraction, the BL class demonstrated a higher $\mathrm{Ml}$, and the $\mathrm{BL}, \mathrm{CL}$, and Her2E classes displayed lower MERs when compared to the HMEC class. d The population doubling level (PDL) over at least 11 weeks in cell culture was determined for each cell line, and the bold lines represent the class averages; both the LumB and Her2E classes exhibited low PDLs. ${ }^{*} p$-value $<0.05$, ${ }^{* *} p$-value that remains significant when controlling for false discovery rate $(5 \%)$ 
Table 1. Predictive relationships between cell cycle growth phases based on cell proliferation markers

\begin{tabular}{llccl}
\hline $\begin{array}{l}\text { Cell line } \\
\text { class }\end{array}$ & S:MI & S:MER & MER:Ml & $\begin{array}{l}\text { Significant } \\
\text { predictive } \\
\text { relationships }\end{array}$ \\
\hline HMEC & $<0.0001^{* *}$ & $0.006^{* *}$ & $0.0120^{*}$ & $\begin{array}{l}\text { S } \rightarrow \text { MER, MER } \rightarrow \text { Ml, } \\
\text { and S } \rightarrow \text { Ml }\end{array}$ \\
BL & 0.5996 & 0.5954 & 0.1659 & N/A \\
LumB & $0.0041^{*}$ & 0.4769 & $<0.0001^{* *}$ & MER $\rightarrow$ Ml, S $\rightarrow$ Ml \\
CL & 0.4827 & 0.3758 & $0.0051^{*}$ & MER $\rightarrow$ MI \\
Her2E & 0.0706 & 0.3090 & 0.1309 & N/A \\
\hline
\end{tabular}

Breast cancer cell lines exhibit impaired cell cycle regulation and $S / G_{2} / M$ progression kinetics. Associations between the S:MI, S:MER, and MI:MER growth parameters were assessed for each class. Significant correlations are shown in the right column. Only the HMEC class showed significant associations among all three parameters; all breast cancer cell line classes exhibited aberrant progression kinetics for at least one cell cycle phase transition. ${ }^{*} p$-value $<0.05,{ }^{* *} p$-value that remains significant when controlling for false discovery rate $(5 \%)$

Due to the variable range of cohesion defect severity, metaphases were examined via a high sensitivity fluorescence in situ hybridization (FISH) assay utilizing a probe targeting the centromere of chromosome 9 (CEP9) to confirm the presence of cohesion defects and evaluate severity in the BL and CL classes. In diploid metaphase cells with properly cohered sister chromatids, the centromeres of the sister chromatids are so close together that they appear to be a single CEP9 locus upon FISH staining. However, in cells exhibiting cohesion defects, the centromeric regions of the sister chromatids are separated and exhibit a doublet pattern containing two visually distinct CEP9 loci. Degrees of cohesion defects were defined as mild, moderate, or severe, ${ }^{42}$ and examples are shown in Fig. $4 \mathrm{~b}$ with white arrows indicating each type of cohesion defect. The $\mathrm{BL}$ and $\mathrm{CL}$ classes both exhibited increases in the total percentage of cells with cohesion defects, suggesting that these classes may experience difficulty completing DNA replication and/or partitioning sister chromatids equally into daughter cells. Furthermore, distributions of cohesion defect severity in both classes also differed when compared to the HMECs, suggesting that an increased number of moderate/severe cohesion defects were found in these classes (Fig. 4b, lower panel). In addition, both the $\mathrm{BL}$ and $\mathrm{CL}$ classes exhibited significantly higher percentages of cells with aneuploidy when compared to HMECs (>4 CEP9 foci per metaphase), suggesting that cohesion defects may lead to inappropriate segregation of duplicate chromosomes during cytokinesis and confirming the presence of genomic instability in these classes (Fig. S2 and Table S3B).

Because the MERs of the $C L$ and $B L$ classes were low compared to the HMEC class (Fig. 3c), the presence of cohesion defects (Fig. $4 \mathrm{a}, \mathrm{b}$ ) have been previously shown to activate the $S A C^{43}$ and severe aneuploidy was observed in both classes (Table S5B, Fig. S2), it was possible that the $\mathrm{BL}$ and $\mathrm{CL}$ classes harbored $\mathrm{SAC}$ defects. Thus, the number of mitotic cells that accumulated over a $24 \mathrm{~h}$ period in the presence/absence of colcemid were measured by flow cytometry. ${ }^{19,} 44$ This assay allows us to distinguish between cell lines that exhibit a low MER due to slow movement through $\mathrm{S}$ and/or $\mathrm{G}_{2}$ phase (appreciable levels of mitotic accumulation in the presence of colcemid appear over $24 \mathrm{~h}$ ) and cell lines that lack a functional SAC (no mitotic accumulation due to an inability to arrest in colcemid). Example flow cytometry profiles for MCF10As (HMEC) and MDA-MB-468s (BL) are shown in the left panel of Fig. 4c. Addition of $100 \mathrm{ng} / \mathrm{mL}$ colcemid dramatically increased the number of mitotic cells in the MCF10As, whereas the number of mitotic cells decreased in the MDA-MB- 468s (Fig. 4c, middle panels). The average increase in mitotic index for each cell line grouped by intrinsic molecular subtype is shown for three independent experiments (right panel, Fig. 4c). Individual cell line averages are provided in Table S4. Both the BL and the Her2E classes appeared to exhibit a defective SAC, and LMM analysis confirmed that SAC function was reduced in the BL class. Although one BL cell line (SUM149) exhibited some mitotic accumulation in the presence of colcemid, this result is likely due to the presence of a subpopulation of $C L$ cells found in this cell line. ${ }^{34}$ Taken together, these data suggest that the combined cohesion defects and aberrant SAC function identified in the BL class may contribute to the high levels of genomic instability observed in BL breast cancers. The presence of an effective SAC in the $C L$ class suggests that cohesion defects in these cell lines are capable of activating the SAC and that the severe aneuploidy observed is likely due to an alternative mechanism of genomic instability. $\left({ }^{*} p<0.05,{ }^{* *} p<0.05, \mathrm{FDR}<0.05\right)$

A decatenation $\mathrm{G}_{2}$ checkpoint gene expression signature correlates with clinical outcomes of breast cancer patients

To determine whether the checkpoint defects identified in the cell line panel were associated with clinical outcomes, a gene expression signature of decatenation $G_{2}$ checkpoint function was identified from the cell line panel using quantitative trait analysis (QTA) (Table S5). This signature was applied to the METABRIC clinical data set, which contains approximately 2000 breast tumor samples that include clinical annotations, intrinsic molecular subtyping information, and gene expression data. ${ }^{45}$ In a univariate analysis, the decatenation $\mathrm{G}_{2}$ checkpoint signature was significantly associated with better overall survival in all breast tumors (HR: 0.7391, Cl: 0.5759-0.9486, log rank $p=0.0176$, Fig. 5a). In a multivariate analysis including the intrinsic molecular subtypes, the checkpoint signature was not significantly associated with overall survival (HR: $0.76843, \mathrm{Cl}$ : 0.5769-1.023, log rank $p=0.071673$ ), but was close to significant.

The decatenation $G_{2}$ checkpoint signature was also predictive of OS outcomes in LumA patients (HR: $0.6039, \mathrm{Cl}: 0.4559-0.7998, p$ $=0.01741$, Fig. $5 \mathrm{~b}$ ), suggesting that impaired decatenation $\mathrm{G}_{2}$ checkpoint function may contribute to poor outcomes in the LumA subtype. The median decatenation $\mathrm{G}_{2}$ checkpoint signature for all breast tumors varied among the subtypes $(p=1.73 \mathrm{e}-133$, Fig. 5c) with the LumB subtype expressing the lowest median checkpoint signature; this observation was independently verified using the UNC337 data set $\left(p=8.46 \mathrm{e}-20\right.$, Table S6). ${ }^{27}$ Although the decatenation $G_{2}$ checkpoint signature was not significantly associated with outcomes within the LumB subtype $(p=0.597$, Table S6), this may be due to the inherently low median decatenation $G_{2}$ checkpoint signature within this subtype. For example, if the majority of LumB tumors are defective for decatenation $G_{2}$ checkpoint function, there may not be enough variation of the signature within the LumB subtype to predict outcomes. Taken together, these data imply that the decatenation $G_{2}$ checkpoint may be a clinically relevant target for the treatment of LumA and/or LumB breast cancer patients.

\section{DISCUSSION}

Although several studies have focused on cell cycle control of human breast cancers and breast cancer cell lines at the $G_{1} / S$ transition, only a few have examined $\mathrm{G}_{2} / \mathrm{M}$ checkpoints in multiple cell lines. ${ }^{46,}{ }^{47}$ However, these studies didn't address DNA damage $G_{2}$ or decatenation $G_{2}$ checkpoint function, nor did they consider the intrinsic molecular subtype of the cell lines. Therefore, the well-characterized panel of breast cancer and HMEC cell lines described in this work provides a valuable resource summarizing cell cycle checkpoint deregulation and genomic instability patterns observed in a breast cancer cell line model system (Table 2). 


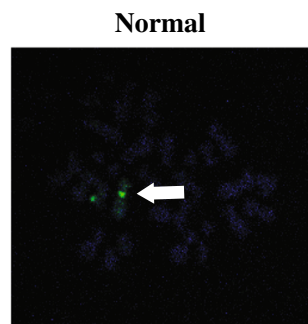

Moderate Cohesion Defect

A

R-HMEC-E
(HMEC)

SUM149

(BL)
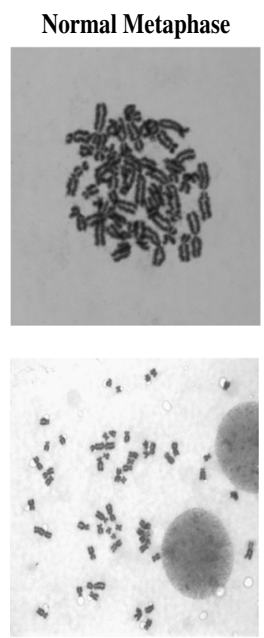

MDA-MB-436

(CL)

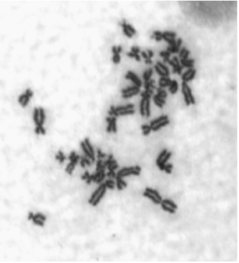

Cohesion Defect
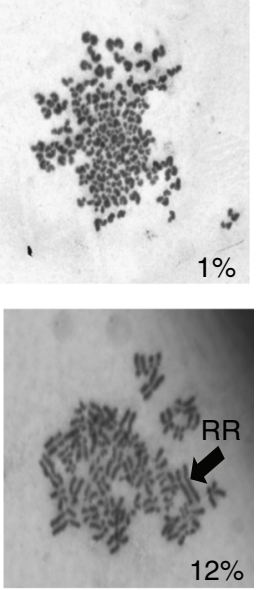

$12 \%$

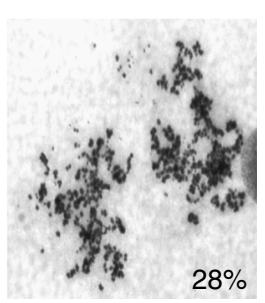

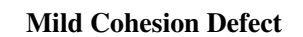

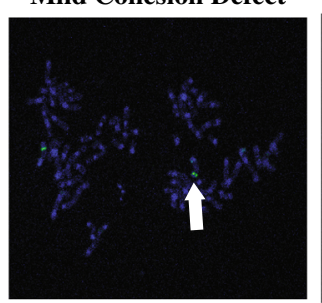

Severe Cohesion Defect
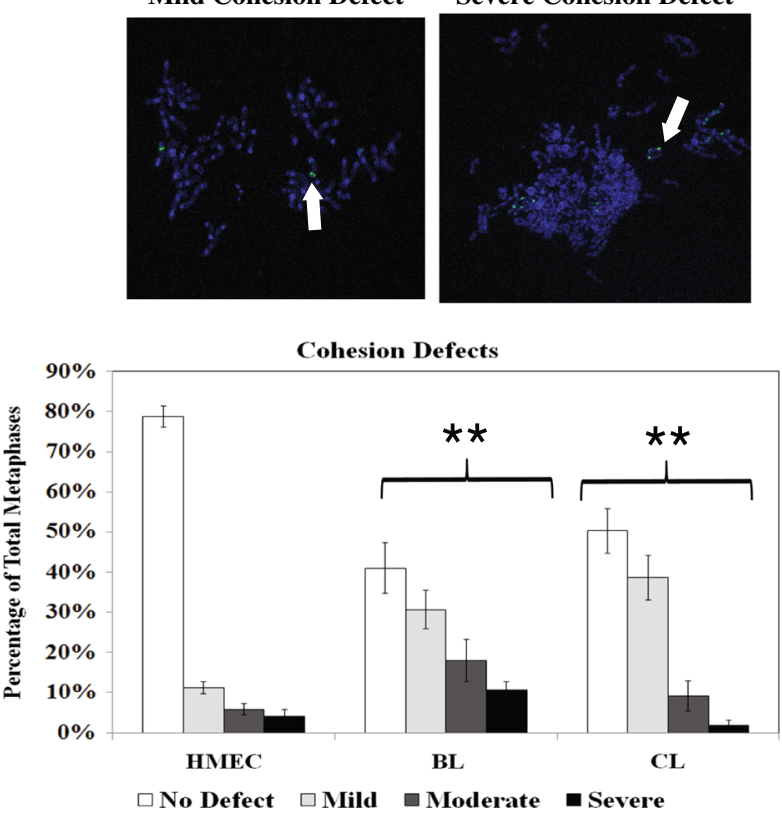

C
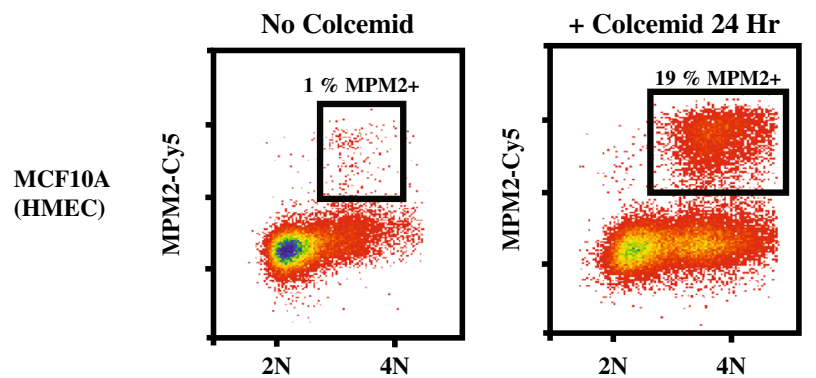

MCF10A (HMEC)
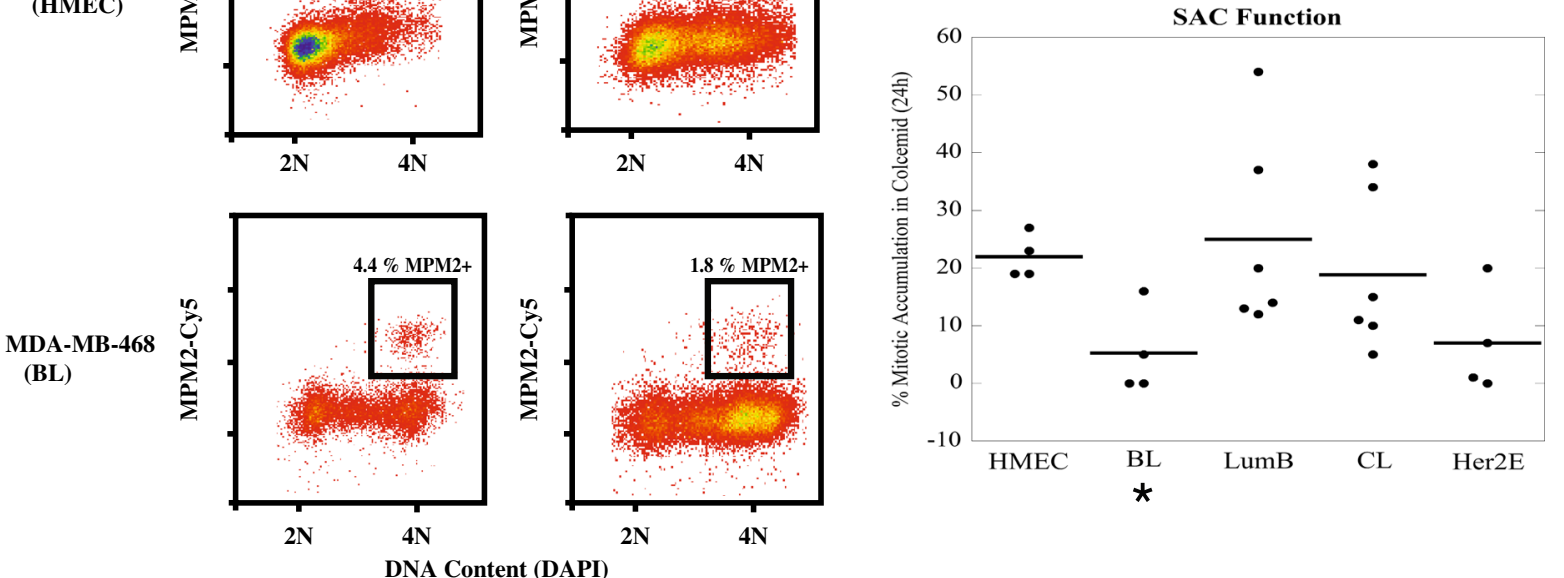

Fig. 4 The BL and CL cell line classes exhibit chromatid cohesion defects; the BL class also harbors a defective SAC. a Examples of cohesion defects observed in metaphase. The percentages of cells containing cohesion defects is shown for each cell line in the lower right corner of the cohesion defect picture. A "railroad" (RR) chromosome lacking a centromeric constriction point is designated with a black arrow in the SUM149 cell line to exemplify a mild cohesion defect. A severe cohesion defect is exemplified by the complete discohesion of the MDA-MB436 metaphase. b Representative examples of the severity of cohesion defects observed during FISH analysis (upper panels). White arrows designate the centromere used for cohesion defect classification. Quantification of cohesion defects (lower panel) demonstrate that both the $\mathrm{BL}$ and $\mathrm{CL}$ classes exhibited variation in the distribution of chromatid cohesion defect severity when compared to the HMECs. Results shown were obtained from at least four different biological replicates for each subtype. c Flow cytometry examples reflecting SAC function in an HMEC line (MCF10A) and a BL breast cancer line (MDA-MB-468). Individual cell line averages obtained from at least three independent experiments are shown with bold lines representing the class average; the BL class exhibited a decrease in SAC function (right panel). ${ }^{*} p$-value $<0.05,{ }^{* *} p$-value that remains significant when controlling for false discovery rate $(5 \%)$ 
A

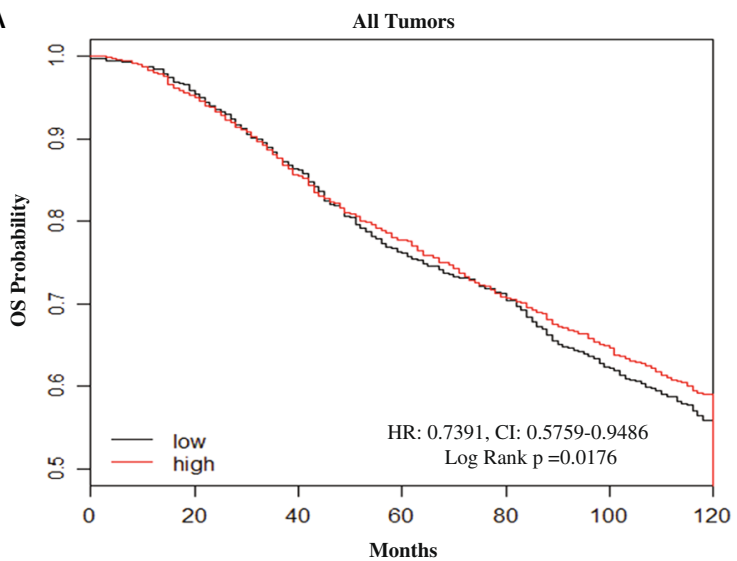

B
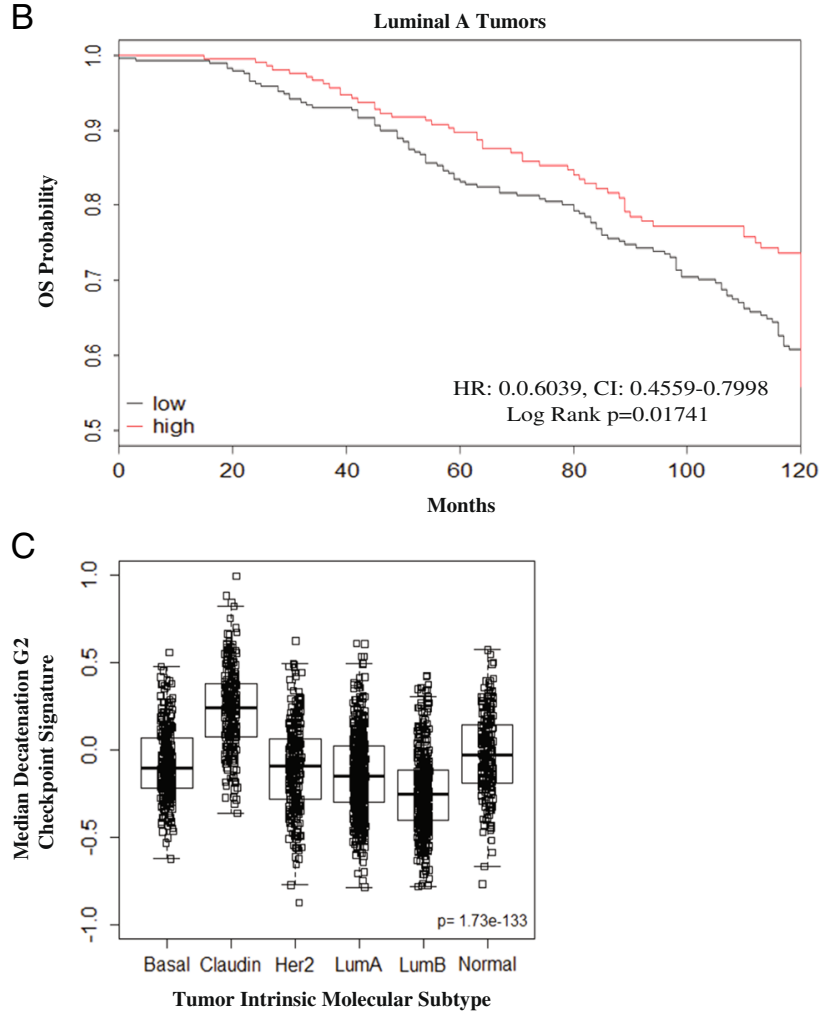

Fig. 5 A decatenation $G_{2}$ checkpoint gene expression signature correlates with clinical outcomes of breast cancer patients. a gene expression signature that positively correlated with decatenation $\mathrm{G}_{2}$ checkpoint function in the breast cancer cell line panel was identified and its relationship with clinical outcomes in the METABRIC study was assessed using a Cox proportional hazards model. A high expression of the decatenation $G_{2}$ checkpoint signature was associated with better overall survival (OS) in all breast tumors ( $\log$ rank $p=0.0176$ ). b For the LumA subtype of breast tumors, high expression of the decatenation $G_{2}$ checkpoint was associated with better OS outcomes (Log Rank $p=0.01741)$. c Median decatenation $G_{2}$ checkpoint signature varies with intrinsic molecular subtype; LumA and LumB breast tumors exhibited the lowest median expression of the decatenation $G_{2}$ checkpoint signature. HR: hazard ratio, OS: overall survival, $\mathrm{Cl}$ : confidence intervals

The results of this study indicate that intrinsic subtypes are associated with unique combinations of genomic instability patterns including aberrant cell cycle progression kinetics, chromosomal aberrations, and defective $G_{2}$ or $M$ checkpoints. The initial $G_{2} / M$ checkpoint screen revealed that the LumB class exhibited a defect in decatenation $G_{2}$ checkpoint function while remaining capable of activating the DNA damage $\mathrm{G}_{2}$ checkpoint (Fig. 2b, 2c), and the results in Fig. $3 c$ and Table 1 support the use of the MER assay as an appropriate measure of $\mathrm{G}_{2}$ checkpoint function in LumB cell lines. The defect in decatenation $\mathrm{G}_{2}$ checkpoint function was observed in concordance with an attenuation of p-Ser15-p53 upon catalytic topo II inhibition with ICRF-193 (Fig. 2e). Although the rate of TP53 mutation in primary LumB breast tumors is approximately $30 \%,{ }^{48}$ most of the LumB cell lines examined harbored wild-type TP53 (Table S7), but failed to significantly activate p53 upon ICRF-193 treatment while retaining at least partial p53 activation in response to etoposide. These results suggest that although both drugs share the same target (topo II), they elicit distinct signaling responses in the LumB class and provide further support for the $G_{2}$ checkpoint results (Fig. 1b).

Decatenation $\mathrm{G}_{2}$ checkpoint defects have also been observed in lung, bladder, melanoma, and colon cancer cell lines. ${ }^{37,}$ 49-51 Two reports have also demonstrated that decatenation $G_{2}$ checkpointdefective melanoma and colon cancer cell lines are hypersensitive to PI3-kinase inhibitors and topo II catalytic inhibitors, respectively. ${ }^{51,52}$ Because the decatenation $G_{2}$ checkpoint response is molecularly distinct from the DNA damage $G_{2}$ checkpoint and the $S A C, 1,16,17$ and the gene expression signature of decatenation $G_{2}$ checkpoint function identified in this study is associated with OS outcomes in breast cancer patients (Fig. 5, Table S6), it may be feasible to induce synthetic lethality in tumors exhibiting defects in decatenation $G_{2}$ checkpoint function with pharmacological agents that catalytically inhibit topo II activity, providing an alternative strategy for targeted drug design to treat poor prognosis LumB breast cancers and a subset of LumA breast cancers. Potential agents that may enhance the cytotoxicity of decatenation $\mathrm{G}_{2}$ checkpoint-defective tumor cells include dexrazoxane, an FDA-approved topo II catalytic inhibitor currently used to prevent anthracycline-induced cardiotoxicity, and PI3 kinase inhibitors that are currently in clinical trials for multiple solid tumors.

The low MERs of the BL and CL classes (Table S2 and Fig. 3c) and insignificant correlations between S:MER and MER:MI (Table 1) prompted the examination of metaphases to assess underlying mechanisms that might lead to altered $S / G_{2} / M$ progression. Both classes displayed increased levels of cohesion defects and aneuploidy (Fig. 4 and Fig. S2); however, only the BL class failed to activate a SAC response (Fig. $4 \mathrm{C}$ ). Although a recent report has suggested that two SAC markers are activated in response to Docetaxel in BL cell lines, functional checkpoint assays were not performed suggesting that aberrant activation of those SAC markers may not be the underlying cause of SAC dysfunction. ${ }^{53}$ The significance of these results is further underscored by studies demonstrating that an effective SAC is required for both paclitaxel and anthracyclines to exert cytotoxic effects, and that in the absence of an effective SAC these drugs induce ploidy increases. ${ }^{22,} 23$ These data suggest that the high incidence of cohesion defects coupled with a defective SAC may promote genomic instability, contribute to high relapse rates, and represent a potential synthetic lethal target for BL breast tumors. ${ }^{22,23,43,54}$

Similar to the BL class, the CL cell lines exhibited a lower MER (Fig. 2c) and an increase in the presence of cohesion defects; however, the $\mathrm{CL}$ class maintained an intact SAC (Fig. 3a, b) suggesting that an alternative mechanism of genomic instability may be present in this class of cell lines. Curiously, the CL class exhibited similar PDL levels, S, MI, and SAC function in comparison to HMECs (Fig. 3a-d, 4d); however, the lower MERs of this class suggest that these cells may transit through $S$ or $G_{2}$ at a slower rate. Although it is presently unclear what effects sister chromatid cohesion defects may have in the $\mathrm{CL}$ subtype, similar mild cohesion defects are associated with genomic instability and the inactivation of MRE-11, SMC1, and CDC4/FBXW7 in colorectal 
stochastic activation of signaling networks that can promote heterogeneity in tumor cells and stem cells. ${ }^{55-57}$ Such heterogeneity in cell cycle checkpoint activation may affect chemotherapy response, induce a hyper-mutagenic tumor microenvironment, and/or possibly contribute to cancer stem cell maintenance.

This is the first report to identify intrinsic subtype-associated cell cycle checkpoint deregulation in breast cancer cell lines and a potentially clinically relevant role for the decatenation $G_{2}$ checkpoint. In addition, this work has provided a summary of $\mathrm{S}$ / $\mathrm{G}_{2} / \mathrm{M}$ progression kinetics, genomic instability mechanisms, and $\mathrm{G}_{2} / \mathrm{M}$ checkpoint defects associated with breast cancer subtypes in a large cell line panel; these characteristics are often difficult to predict by gene expression data alone due to the highly dynamic nature of checkpoints, which often rely on kinase/phosphatase activity and protein degradation pathways to initiate/sustain checkpoint activation. In summary, the subtype-associated genomic instability phenotypes described in this work may be useful for the development of new targeted therapies for the LumB, BL, and $C L$ subtypes, may help identify biomarkers to serve as predictive indicators of therapeutic response, and may explain the underlying causes of differential patient responses to current chemotherapeutic regimens.

-Delayed progression of $\mathrm{S}$ and/or G2 phase

-Aneuploidy•Increased percentages of cohesion defects

-Functional SAC

Her2E

-Attenuated p-Ser15 p53 activation in response to catalytic topo II inhibitor

- Low proliferation levels (S, MER, PDL)

-Aberrant regulation of $S / G 2 / M$ progression -Aneuploidy

Note: A summary of growth characteristics, $\mathrm{G}_{2} / \mathrm{M}$ checkpoint function, and genomic instability patterns associated with each intrinsic subtype of breast cancer cell lines

cancers $^{42}$ and future studies will attempt to further characterize S/ $\mathrm{G}_{2} / \mathrm{M}$ cell cycle progression defects in the $\mathrm{CL}$ subtype.

Components of the cell cycle regulatory machinery and checkpoint function affect the response to chemotherapy in breast cancer cells. ${ }^{24}$ Due to the increasing utilization of breast cancer intrinsic molecular subtypes in clinical settings for prognostic purposes and chemotherapeutic treatment guidance, the identification of subtype-associated genomic instability trends in a standardized panel of in vitro cell lines may contribute to the development of diagnostic assays, novel targeted drug therapies, and new personalized chemotherapy regimens. For example, drugs that target aberrant cell cycle control at the $G_{0} / G_{1}$ restriction point such as the cdk4/6 inhibitor Palbociclib ${ }^{\circledR}$ have recently been approved by the FDA to treat hormone therapy-resistant $\mathrm{ER}^{+}$ tumors and are currently being exploited in the clinic to induce synthetic lethality in cancers exhibiting deregulation of the RB/ cyclin D1 pathway. ${ }^{25}$ Although we attempted to identify subtypespecific genetic mutations in the breast cancer cell line panel that might contribute to the observed genomic instability patterns and identify potential synthetic lethal targets, the resulting data analysis failed to reveal subtype-specific mutations (Fig. S3 and Table S8), similar to published results for primary breast tumors. ${ }^{48}$

The significance of the subtype-associated genomic instability mechanisms outlined herein is further enhanced by the observation that intrinsic molecular subtypes can often indicate an intermediate phenotype; thus, the use of functional $\mathrm{G}_{2} / \mathrm{M}$ checkpoint assays or other measures of genomic instability to complement current clinical determinants of chemotherapeutic response may help relieve some of the ambiguity surrounding these "borderline" tumors. We speculate that hetreogeneous activation of cell cycle checkpoints would likely result in stochastic bypass of these checkpoints leading to inappropriate initiation of DNA synthesis and/or precocious entry into mitosis, similar to the

\section{METHODS}

\section{Cell line panel}

At least four cell lines representing each of the intrinsic molecular subtypes were assayed. Growth medium, conditions, and cell line source are detailed in Table S7. Periodic tests for mycoplasma contamination using a commercial kit were negative. PAM50 subtype calls were based on microarray gene expression data. ${ }^{34}$

\section{Cell proliferation markers}

Cells were plated (day 0), fed (day 2), and treated (day 3) with $10 \mu \mathrm{M} \mathrm{EdU}$ for $2 \mathrm{~h}$ and fixed. Samples were stained with: MPM2-Cy5, Click-It EdU-Alexa 488 substrate, and DAPI (See Table S9 for catalog numbers). All samples were analyzed using a Beckman Coulter Dako CyAn ADP Analyzer at the UNC Flow Cytometry Core Facility. At least 30,000 cells were analyzed per sample and percentages of mitotic cells (MPM2 $2^{+} / 4$ N DNA content) or EdU ${ }^{+}$ cells ( $S$ phase) were quantified. Results were obtained from at least three independent experiments for each cell line.

\section{Population doubling level (PDL)}

Cells were passaged approximately once a week and fed every 2-3 days with fresh medium. The total number of cells recovered was determined using a Beckman Coulter Counter Z1 instrument and PDL was calculated as follows: $P D L=[\log$ (total number of cells recovered/total number of cells plated)]/log2. PDL/week was calculated by determining the number of PDLs occurring over an average 7 day period.

\section{Flow cytometry MER assay}

On day 3, cells were treated with $100 \mathrm{ng} / \mathrm{mL}$ colcemid and $0.1 \%$ DMSO, 4 $\mu \mathrm{M}$ ICRF-193, or $4 \mu \mathrm{M}$ etoposide for $0,2,4$, and $6 \mathrm{~h}$. MPM2-Cy5/DAPI of cells in mitosis was calculated by regression of the linear portion of the resulting line (2-6h time points) and the slope of that line was used to quantify MER (\% of cells entering mitosis/h) as previously described. ${ }^{36}$ Results were obtained from at least three independent experiments for each cell line.

\section{Western immunoblotting}

Standard immunoblotting techniques were used to detect phosphorylated and total proteins as previously described. ${ }^{1}$ Total and phosphorylated proteins were semi-quantified using Image J software v. $1.45(\mathrm{NIH})$ (Table S9-antibody list). Pixel intensities of each phosphorylated protein were normalized to a-tubulin. The increment of increase in protein levels for the drug-treated samples compared to DMSO samples were used to measure protein activation. Values were subsequently normalized to the increment measured for the same NHF1-hTERT positive control sample as staining of mitotic cells was performed. The rate of change in the percent 
an internal normalization control to facilitate comparisons among different western blots and account for different exposures. Western blots shown are representative of two independent experiments.

\section{Spindle assembly checkpoint assay}

Cells were incubated with $100 \mathrm{ng} / \mathrm{mL}$ colcemid or $0.1 \%$ DMSO (vehicle control) for 0 or $24 \mathrm{~h}$ and then fixed, stained, and quantified as described for the MER assay. Results were obtained from at least three independent experiments for each cell line.

\section{Cytogenetic studies}

Metaphases were prepared as previously described, ${ }^{1}$ and examined using an Olympus BH2 microscope (100X objective) with a SPOT RT camera. At least 100 metaphases were scored for each treatment group/cell line when available-if fewer than 100 metaphase cells were present, all metaphases present were analyzed. All analyses were performed blinded to sample identity.

\section{Fluorescence in situ hybridization (FISH)}

FISH samples were prepared as previously described. ${ }^{1}$ At least 100 metaphases were scored per cell line when available-if fewer than 100 metaphase cells were present, all metaphases present were analyzed. All analyses were performed blinded to sample identity on a Zeiss LSM 700 Confocal Laser Scanning Microscope (UNC-CH Microscopy Services Laboratory). The severity of cohesion defects was assessed based on a previous study. ${ }^{42}$ Metaphases containing more than one type of cohesion defect were categorized as exhibiting the most severe cohesion defect.

\section{QTA and clinical outcomes analysis}

Cell line gene expression data were obtained from Dr. Katherine Hoadley and Dr. Charles M. Perou at the University of North Carolina-Chapel Hill and is publicly available. ${ }^{34}$ Array data were Lowess normalized and expressed as log2 ratios. Of the 24 cell lines comprising the panel, 22 were present in the microarray dataset (R-HMEC-E and BT20 were unavailable). QTA was performed using the significance analysis of microarrays (SAM) package in $\mathrm{R}^{58}$ Genes that significantly correlated with a quantitative trait (such as \% Inhibition of MER in the presence of ICRF-193 to represent decatenation $G_{2}$ checkpoint function) were selected to generate the gene signature using the QTA tool provided within the SAM package at a low FDR $(\leq 0.1) .^{59}$ These gene lists were subsequently applied to an independent set of publically available clinical data containing overall survival data, clinical annotations, PAM50 subtyping assignments, and gene expression data for approximately 2000 breast cancer patients. ${ }^{45}$ Clinical outcome association analyses were performed using a Cox proportional hazards model in the R survival package.

\section{Statistical analyses}

A set of LMMs were developed to compare the intrinsic subtype classes with the HMEC class. All of the results presented in this manuscript are supported by sensitivity analyses performed using the same data set after removal of one or more highly influential data points (based on the wellknown Cook's distance metric). If the statistical conclusions were the same in both the primary and sensitivity analyses, it was concluded that the particular observation did not unduly influence the analysis and the primary analysis result was reported as statistically significant. All $p$-values, $95 \%$ confidence intervals, and details regarding the specific statistical models employed for each individual analysis are included in the supplementary information (SI). ${ }^{*} p$-value $<0.05,{ }^{* *} p$-value that remains significant when controlling for false discovery rate (5\%).

\section{ACKNOWLEDGEMENTS}

We thank Dr. Bernard Omolo and Linda Chaba for assistance with gene expression data analysis. We also thank Dr. Matthew Hirsch and Dr. Marila Cordeiro-Stone for their thoughtful comments. This work was supported by an $\mathrm{NIH}-\mathrm{NCl}$ Ruth $\mathrm{L}$. Kirschstein National Research Service Award Grant \# 1F32CA134155-01 (J.J. Bower), PHS grants CA81343 and P30-ES10126 (W.K. Kaufmann), NCI Breast SPORE program P50CA58223-09A1 (C.M. Perou), and by the Breast Cancer Research Foundation (C. M. Perou). The UNC Flow Cytometry Core Facility is supported in part by an $\mathrm{NCl}$ Center Core Support Grant (P30CA016086) to the UNC Lineberger Comprehensive Cancer Center.

\section{AUTHOR CONTRIBUTIONS}

J.J.B. and W.K.K. designed the study and wrote the manuscript. J.J.B., L.D.V., and S.S-R. performed the experiments with logistical support from D.A.S. M.P. and J.G.I. designed and performed the statistical analyses for the cell line data. C.M.P. and K.A $\mathrm{H}$. provided cell lines, subtype classifications, and guidance for microarray and clinical data analyses.

\section{COMPETING INTERESTS}

C.M.P. is an equity stock holder and member of the Board of Directors of BioClassifier LLC and GeneCentric Diagnostics. C.M.P. is also listed as an inventor on a patent application on the PAM50 molecular assay. The remaining authors declare that they have no competing interests.

\section{REFERENCES}

1. Bower, J. J. et al. Topoisomerase Ilalpha maintains genomic stability through decatenation G(2) checkpoint signaling. Oncogene. 29, 4787-4799, doi:10.1038/ onc.2010.232 (2010).

2. Taylor, S. S. \& McKeon, F. Kinetochore localization of murine bub1 is required for normal mitotic timing and checkpoint response to spindle damage. Cell $\mathbf{8 9}$, 727-735 (1997)

3. Hartwell, L. H. \& Kastan, M. B. Cell cycle control and cancer. Science 266, 1821-1828 (1994).

4. Pardee, A. B. G1 events and regulation of cell proliferation. Science 246, 603-608 (1989).

5. Weinberg, R. A. The Retinoblastoma protein and cell cycle control. Cell 81, 323-330 (1995).

6. Marie Classon, E. H. The retinoblastoma tumor suppressor in development and cancer. Nat. Rev. Cancer 2, 910-917 (2002).

7. Daniel Menendez, A. I. \& Michael A. Resnick. The expanding universe of p53 targets. Nat. Rev. Cancer 9, 724-737 (2009).

8. Brown, C. J., S. L., Verma, C. S., Fersht, A. R. \& Lane, D. P. Awakening guardian angels: drugging the p53 pathway. Nat. Rev. Cancer 9, 862-873 (2009).

9. Agarwal, M. L., Agarwal, A., Taylor, W. R. \& Stark, G. R. p53 controls both the G2/M and the $\mathrm{G} 1$ cell cycle checkpoints and mediates reversible growth arrest in human fibroblasts. Proc. Natl. Acad. Sci. U.S.A. 92, 8493-8497 (1995).

10. Hartwell, L. H. \& Weinert, T. A. Checkpoints: controls that ensure the order of cell cycle events. Science 246, 629-634 (1989).

11. Deming, P. B. et al. The human decatenation checkpoint. Proc. Natl. Acad. Sci. U.S.A. 98, 12044-12049, doi:10.1073/pnas.221430898 (2001).

12. Downes, C. S. et al. A topoisomerase Il-dependent G2 cycle checkpoint in mammalian cells. Nature. 372, 467-470 (1994).

13. Nurse, P. \& Bissett, Y. Gene required in G1 for commitment to cell cycle and in G2 for control of mitosis in fission yeast. Nature. 292, 558 (1981).

14. Nigg, E. A. Cyclin-dependent protein kinases: key regulators of the eukaryotic cell cycle. Bioessays 17, 471-480 (1995).

15. Morgan, D. O. Principles of CDK regulation. Nat. Cell. Biol. 374, 131-134 (1995).

16. Luo, K., Yuan, J., Chen, J. \& Lou, Z. Topoisomerase Ilalpha controls the decatenation checkpoint. Nat. Cell. Biol. 11, 204-210, doi:10.1038/ncb1828 (2009).

17. Skoufias, D. A., Lacroix, F. B., Andreassen, P. R., Wilson, L. \& Margolis, R. L. Inhibition of DNA decatenation, but not DNA damage, arrests cells at metaphase. Mol. Cell. 15, 977-990, doi:10.1016/j.molcel.2004.08.018 (2004).

18. Xu, B., Kim, S.-T. \& Kastan, M. B. Involvement of Brca1 in S-Phase and G2-Phase checkpoints after ionizing irradiation. Mol. Cell. Biol. 21, 3445-3450 (2001).

19. Kung, A. L., Sherwood, S. W. \& Shimke, R. T. Cell line-specific differences in the control of cell cycle progression in the absence of mitosis. Proc. Natl. Acad. Sci. U.S.A. 87, 9553-9557 (1990).

20. Rui-Hong Wang, H. Y., Chu-Xia Deng. A requirement for breast-cancer-associated gene 1 (BRCA1) in the spindle checkpoint. Proc. Natl. Acad. Sci. 101, 17108-17113 (2004).

21. Musacchio, A. The molecular biology of spindle assembly checkpoint signaling dynamics. Curr. Biol. 25, R1002-R1018 (2015).

22. Vogel, C., Kienitz, A., Muller, R. \& Bastians, H. The mitotic spindle checkpoint is a critical determinant for topoisomerase-based chemotherapy. J. Biol. Chem. 280, 4025-4028, doi:10.1074/jbc.C400545200 (2005).

23. Sudo, T., Nitta, M., Saya, H. \& Ueno, N. T. Dependence of paclitaxel sensitivity on a functional spindle assembly checkpoint. Cancer Res. 64, 2502-2508 (2004).

24. Bosco, E. E. et al. The retinoblastoma tumor suppressor modifies the therapeutic response of breast cancer. J. Clin. Invest. 117, 218-228, doi:10.1172/JCI28803 (2007). 
25. Cadoo, K. A., Gucalp, A. \& Traina, T. A. Palbociclib: an evidence-based review of its potential in the treatment of breast cancer. Breast Cancer 6, 123-133, doi:10.2147/BCTT.S46725 (2014).

26. Perou, C. M. et al. Molecular portraits of human breast tumors. Nature. 406, 747-752 (2000)

27. Prat, A. et al. Phenotypic and molecular characterization of the claudin-low intrinsic subtype of breast cancer. Breast. Cancer Res. 12, R68, doi:10.1186/ bcr2635 (2010).

28. Santarpia, L. et al. DNA repair gene patters as prognostic and predictive factors in molecular breast cancer subtypes. Oncologist. 18, 1063-1073 (2013).

29. Docquier, A. et al. The transcriptional coregulator RIP140 represses E2F1 activity and discriminates breast cancer subtypes. Clin. Cancer Res. 16, 2959-2970, doi:10.1158/1078-0432.CCR-09-3153 (2010).

30. Sorlie, T. et al. Gene expression patterns of breast carcinomas distinguish tumor subclasses with clinical implications. Proc. Natl. Acad. Sci. U. S. A. 98 , 10869-10874, doi:10.1073/pnas.191367098 (2001).

31. Hastak, K., Alli, E. \& Ford, J. M. Synergistic chemosensitivity of triple-negative breast cancer cell lines to poly(ADP-Ribose) Polymerase inhibition, gemcitabine, and cisplatin. Cancer Res. 70, 7970-7980 (2010).

32. Heiser, L. M. et al. Subtype and pathway specific responses to anticancer compounds in breast cancer. Proc. Natl. Acad. Sci. U. S. A. 109, 2724-2729, doi:10.1073/pnas.1018854108 (2012).

33. Neve, R. M. et al. A collection of breast cancer cell lines for the study of functionally distinct cancer subtypes. Cancer Cell 10, 515-527, doi:10.1016/j. ccr.2006.10.008 (2006).

34. Prat, A. et al. Characterization of cell lines derived from breast cancers and normal mammary tissues for the study of the intrinsic molecular subtypes. Breast. Cancer. Res. Treat. 142, 237-255, doi:10.1007/s10549-013-2743-3 (2013).

35. Kao, J. et al. Molecular profiling of breast cancer cell lines defines relevant tumor models and provides a resource for cancer gene discovery. PLoS ONE 4, e6146, doi:10.1371/journal.pone.0006146 (2009).

36. Bower, J. J. et al. Revised genetic requirements for the decatenation $\mathrm{G} 2$ checkpoint: the role of ATM. Cell Cycle 9, 1617-1628 (2010).

37. Nakagawa, T. et al. Identification of decatenation G2 checkpoint impairment independently of DNA damage G2 checkpoint in human lung cancer cell lines. Cancer. Res. 64, 4826-4832 (2004).

38. Barretina, J. et al. The cancer cell line encyclopedia enables predictive modelling of anticancer drug sensitivity. Nat. Cell Biol. 483, 603-607 (2012).

39. Schlegel, R. \& Pardee, A. B. Caffeine-induced uncoupling of mitosis from the completion of DNA replication in mammalian cells. Science 232, 1264-1266 (1986).

40. Weinert, T. A. \& Hartwell, L. H. The RAD9 gene controls the cell cycle response to DNA damage in saccharomyces cerevisiae. Science 241, 317-322 (1988).

41. Lee, L. H., Yang, H. \& Bigras, G. Current breast cancer proliferative markers correlate variably based on decoupled duration of cell cycle phases. Sci. Rep. 4, 5122, doi:10.1038/srep05122 (2014).

42. Barber, T. D. et al. Chromatid cohesion defects may underlie chromosome instability in human colorectal cancers. Proc. Natl. Acad. Sci. U.S.A. 105, 3443-3448, doi:10.1073/pnas.0712384105 (2008).

43. de Lange, J. et al. Defective sister chromatid cohesion is synthetically lethal with impaired APC/C function. Nat. Comm. 6, 8399 (2015).

44. Andreassen, P. R., Skoufias, D. A. \& Margolis, R. A. In Checkpoint Controls and Cancer: Activation and Regulation Protocols Vol. 2 Methods in Molecular Biology (ed Axel H. Schonthal) Ch. 15, 213-225 (Humana Press Inc., 2004).
45. Curtis, C. et al. The genomic and transcriptomic architeture of 2000 breast tumours reveals novel subgroups. Nature. 486, 346-352 (2012).

46. Blajeski, A. L., Phan, V. A., Kottke, T. J. \& Kaufmann, S. H. G1 and G2 cell-cycle arrest following microtubule depolymerization in human breast cancer cells. J Clin Invest 110, 91-99, doi:10.1172/jci200213275 (2002).

47. Erson, A. E. \& Petty, E. M. CHFR-associated early G2/M checkpoint defects in breast cancer cells. Mol. Carcinog. 39, 26-33, doi:10.1002/mc.10161 (2004).

48. Network, T. C. G. A. Comprehensive molecular portraits of human breast tumours. Nature. 490, 61-70 (2012).

49. Brooks, K. et al. Defective decatenation checkpoint function is a common feature of melanoma. J. Invest. Dermatol. 134, 150-158, doi:10.1038/jid.2013.264 (2014).

50. Doherty, S. C. et al. Cell cycle checkpoint function in bladder cancer. J. Natl. Cancer. Inst. 95, 1859-1868 (2003).

51. Jain, C. K., Roychoudhury, S. \& Majumder, H. K. Selective killing of G2 decatenation checkpoint defective colon cancer cells by catalytic topoisomerase II inhibitor. Biochim. Biophys. Acta. 1853, 1195-1204 (2015).

52. Brooks, K. et al. Decatenation checkpoint defective melanomas are dependent on PI3K for survival. Pigment Cell Melanoma Res. 27, 813-821 (2014).

53. Kung, P. P. et al. Chemo-genetic evaluation of the mitotic kinesin CENP-E reveals a critical role in triple-negative breast cancer. Mol. Cancer. Ther. doi:10.1158/15357163.MCT-14-0083-T (2014).

54. Carey, L. A. et al. The triple negative paradox: primary tumor chemosensitivity of breast cancer subtypes. Clin. Cancer. Res. 13, 2329-2334, doi:10.1158/1078-0432. CCR-06-1109 (2007).

55. Jinho Lee, A. T., Shum, Victor, Mills, GordonB., Mancini, MichaelA. \& Igoshin, OlegA. Gabor Balazsi. Unraveling the regulatory connections between two controllers of breast cancer cell fate. Nucleic. Acids. Res. 42, 6839-6849 (2014).

56. Jiyoung Lee, J. L., Kevin S. Farquhar, Jieun Yun, Casey A. Frankenberger, Elena Befilacqua, Kam Yeung, Eun-Jin Kim, Gabor Balazsi, Marsha Rich Rosner. Network of mutually repressive metastasis regulators can promote cell heterogeneity and metastatic transitions. Proc. Natl. Acad. Sci. U.S.A. 111, E364-E373 (2014).

57. Singh, A. M. Cell cycle-driven heterogeneity: on the road to demystifying the transitions between "poised" and "restricted" pluripotent cell states. Stem. Cells. Int. 1-9 (2015).

58. Team, R. C. R: A language and environment for statistical computing. (2016)

59. Tusher, V. G., Tibshirani, R. \& Chu, G. Significance analysis of microarrays applied to the ionizing radiation response. Proc. Natl. Acad. Sci. U.S.A. 98, 5116-5121 (2001).

\section{c) (i)}

This work is licensed under a Creative Commons Attribution 4.0 International License. The images or other third party material in this article are included in the article's Creative Commons license, unless indicated otherwise in the credit line; if the material is not included under the Creative Commons license, users will need to obtain permission from the license holder to reproduce the material. To view a copy of this license, visit http://creativecommons.org/licenses/by/ $4.0 /$

(c) The Author(s) 2017

Supplementary Information accompanies the paper on the npj Breast Cancer website (doi:10.1038/s41523-017-0009-7). 